\title{
Actividades socio-económicas y los aliados estratégicos en el turismo, ciudad de Babahoyo - Los Ríos
}

\author{
@®@ \\ Socio-economic activities and strategic allies in tourism, city of \\ Babahoyo - Los Ríos
}

Christian Rivera García. ${ }^{1}$, Maricela Izurieta Puente. ${ }^{2}$ \& Jazminia Zuñiga Mayorga. ${ }^{3}$

Recibido: 12-08-2021 / Revisado: 22 -08-2021 /Aceptado: 08-09-2021/ Publicado: 05-12-2021

\begin{abstract}
DOI: https://doi.org/10.33262/concienciadigital.v4i4.2.1978

Introduction. The paralysis of the tourism sector due to the pandemic caused great economic losses in the country, families without sources of income, totally desolate tourist sectors, temporary and permanent closure of many businesses, all this generating great concerns in the tourist services sector. Socio-economic activity are actions of exchange and consumption, which compromise the productivity of a country in conjunction with its goods, services, products and recreational processes, from the field of tourism in general, strategic allies provide the tourism sector with the help that these They require to increase their income, and facilitate the process of attending to tourists, such as science, technology and innovation, necessary and indispensable tools in the desire to provide a full and pleasant quality service. Objective, analyze socio-economic activities to take advantage of the disposition of strategic allies in tourism. Methodology. Application of the types of research: descriptive, exploratory, explanatory and correlational, the descriptive method allowed detail and analyze the socioeconomic activities of the country and the strategic allies linked to this activity, specifying the current situation in its different levels and concepts of commercial positioning as a national tourist subject, the quantitative method led to the number of companies trying to invest in tourist activities in Ecuador. Results. The was asked 160 students with a series of five questions referring to the country's tourist socio-economic activities and its strategic allies. Conclusions. The main economic activity is gastronomy, the public sector
\end{abstract}

\footnotetext{
1 Universidad Técnica de Babahoyo FCJSE, Ecuador. crivera@utb.edu.ec

2 Universidad Técnica de Babahoyo FCJSE, Ecuador. eizurieta@utb.edu.ec

3 Universidad Técnica de Babahoyo FCJSE, Ecuador. jzuniga@ @cjse.utb.edu.ec
} 
that links economic productivity is the Mintur and the private sector presenting attractive investment plans by the Provincial Chamber of Tourism.

Keywords: socio-economic activity, productivity, strategic allies, commercial positioning.

\section{Resumen}

Introducción. La paralización del sector turístico debido a la pandemia ocasiono grandes pérdidas económicas en el país, familias sin fuentes de ingreso, sectores turísticos totalmente desolados, cierre temporal y permanente de muchos negocios, todo esto generando grandes preocupaciones en el sector de servicios turísticos. La actividad socioeconómica son acciones de intercambio y consumo, que comprometen la productividad de un país en conjunto con sus bienes, servicios, productos y procesos recreativos, desde el ámbito del turismo en general los aliados estratégicos brindan al sector turísticos la ayuda que estos requieran para aumentar sus ingresos, y faciliten el proceso de atención a los turistas, como ciencia, tecnología e innovación, herramientas necesarias e indispensables en el deseo de brindar un servicio de calidad plena y placentera. Objetivo, analizar las actividades socioeconómicas para aprovechar la disposición de los aliados estratégicos en el turismo. Metodología. Aplicación de los tipos de investigación: descriptivo, exploratorio, explicativo y correlacional, el método descriptivo permitió, detallar y analizar las actividades socioeconómicas del país y los aliados estratégicos eslabonados para esta actividad, especificando la situación actual en sus diferentes niveles y conceptos de posicionamiento comercial como sujeción turística nacional, el método cuantitativo propició el número de empresas que tratan de invertir en actividades turísticas en Ecuador. Resultados. La se realizó a 160 estudiantes con una serie de cinco preguntas referentes a las actividades socioeconómicas turísticas del país y sus aliados estratégicos. Conclusiones. La principal actividad económica es la gastronómica, el sector publico que enlaza la productividad económica es el Mintur y del sector privado presentando atractivos planes de inversión por la Cámara Provincial de Turismo.

Palabras Clave: actividad socioeconómica, productividad, aliados estratégicos, posicionamiento comercial.

\section{Introducción}

La investigación se enfoca en las actividades socioeconómicas y los aliados estratégicos en el turismo. Este trabajo encaja en la línea de investigación desarrollo de bienes, productos servicios y procesos turísticos y la sublínea de investigación proyecciones para el mejoramiento de la calidad de bienes, productos, servicios y procesos turísticos en la Universidad Técnica de Babahoyo, de la carrera de Hotelería y Turismo. El turismo es una actividad económica y social de gran trascendencia para muchas naciones en el planeta, que se convirtió en una herramienta eficaz para un desarrollo integral e inclusivo, con su papel protagónico en la generación de oportunidades de trabajo y optimizar la 
calidad de vida poblacional de los países turísticos, desarrollo de emprendimientos, infraestructura, divisas de exportación, entre otras.

Han existido considerables logros en la aplicación de política pública en lo que a turismo respecta, con visibles acciones desarrolladas en el último quinquenio, varios elementos del turismo mundial fueron configurando esta nueva fase que añade puntos involucrados estrechamente con la vida diaria de la sociedad, sus riquezas ancestrales, así como el rescate de los saberes y prácticas locales, las cuales tienen que ser considerados en una perspectiva de grupo, que genere propuestas colectivas que generen un mayor impacto en el turismo. Las iniciativas de Política de Turismo en los últimos años, han estado encaminadas a consolidar el turismo como un factor dinamizador del desarrollo económico y social del Ecuador, con la colaboración del sector público bajo la rectoría del Ministerio de Turismo (MINTUR), el involucramiento activo de otras entidades gubernamentales, tales como los gobiernos autónomos descentralizados parroquiales, municipales y metropolitanos, y provinciales; y, la colaboración de los actores del sector privado y comunitario por medio de las Cámaras de Turismo, los Gremios y las Comunidades Locales. La ciudad de Babahoyo cuenta con productividad agrícola de gran variedad que posibilita la inversión nacional y extranjera en sus diferentes niveles económicos de exportación inclusive brindando posibilidad de participación de los actores públicos y privados en conjunto con asesoramiento de profesionales en el área turística y otras disciplinas de apoyo.

El presente trabajo rescata las actividades socioeconómicas desarrolladas en la actividad turística se alcanzará las distintas estrategias que nos ayuden al crecimiento empresarial con ayuda de los aliados estratégicos de las comunidades para mejorar la afluencia de visitantes, ingresos económicos y fuentes de trabajo para la sociedad.

Este proyecto es transcendente porque en el futuro se asocian diferentes aliados estratégicos nacionales e internacionales con inversiones económicas y financieras que posibilitan más fuentes de trabajo y diversidad de proyectos turísticos en la región, multiplicando los bienes, servicios, productos y procesos turísticos, mejorando la calidad de vida de la población y vivir en armonía con el medio ambiente.

Los beneficiarios de este proyecto será la comunidad local, asociación de Cámaras, inversionistas, las entidades que se encuentren vinculadas en el sector turístico de manera directa e indirecta y que permitan mejorar la economía del país, al igual que los turistas quienes obtendrán servicios de mayor calidad y estancia plena y placentera. Es factible porque se trabajará en conjunto con organizaciones como las ONGs,

ONU con promoción al turismo para proyectos sin fines de lucro, y otras organizaciones que permitan buscar nuevas estrategias de promoción y desarrollo turístico del país.

De acuerdo con López (2020) (citado en Guzmán \& Maldonado, 2021):

La pandemia se presenta en una situación débil y sensible desde el punto de la economía. Todo ello se ha producido en un escenario de paralización de las 
cadenas globales de intercambio de bienes y servicios. Esto se une al colapso total del turismo, lo que está frenando una importante fuente de ingresos para los países latinoamericanos. (pág. 32)

Clavitea (2017) afirma que:

Es el elemento consecuencial, siendo la concentración de turistas y las facilidades y servicios inherentes en áreas de destino incrementan una variedad de repercusiones. Las repercusiones económicas abarcan los costos monetarios y beneficios que resultan del desarrollo y uso de facilidades turísticas y servicios; las repercusiones sociales son los cambios en el medio de vida de los residentes de las áreas de destino. (p. 36)

La idea de impacto económico, en este marco, alude al efecto que una medida, una acción o un anuncio generan en la economía. Cuando algo tiene impacto económico, provoca consecuencias en la situación económica de una persona, una comunidad, una región, un país o el mundo (Sosa, 2018, p. 31).

La pandemia a afectado a tres aspectos fundamentales en la cadena de producción creando trastorno en la cadena de suministro y en el mercado afectando con esto a la producción por tal motivo el impacto financiero que tuvieron que afrontar las empresas y los mercados fueron altos y esto depende mucho de la reacción del publico hacia la enfermedad.

Haciendo referencia a la gripe pandémica que existió en el año 2019 los países que se vieron afectado por esta pandemia también aplicaron restricciones y recomendaciones a tiempo sin instituciones farmacéutica porque se pudo controlar a tiempo.

Con el anuncio de la vacuna de varios farmacéuticos se pretende controlar esta pandemia en un cierto tiempo para que la economía de todos los países se reactive y se pueda continuar con normalidad las actividades mercantiles.

\section{Economía social y solidaria}

La Economía Social y Solidaria (ESS) se define como un movimiento político y social promotor de nuevas formas de producción, distribución y consumo, además de ser considerada como un sector de la economía (Piquinela, 2020).

Coraggio (2020) considera "es un modo de hacer economía en función de construir conscientemente, desde la sociedad y el Estado, una sociedad centrada en lazos solidarios, organizando de manera asociada y cooperativa la producción, distribución, circulación y consumo de bienes y servicios" (p.12).

Dávila, L. (2020) expresa que "la economía social y solidaria (ESS) tiene la perspectiva de una economía plural, combinando lógicas económicas variadas (reciprocidad, redistribución y mercado) y es una invitación a rechazar la creciente hegemonía de las lógicas de mercado" (p.248). 
Es una acción económica de atención a los grupos de atención prioritario o grupos vulnerables que busca nuevas formas de generar ingresos a la población con diferentes estrategias de mercadeo basada en el diálogo y en las experiencias que se desarrollan, busca el bienestar de la persona en todo su contexto, teniendo en cuenta que su tiempo vale, es parte de que lo que es la economía solidaria.

Es un método para buscar la nivelación económica de manera colectiva y comunitaria con todos los que en ella participan, creando una mejor calidad de vida y el lucro colectivo, satisfaciendo las necesidades económicas de los participantes y generando nuevos lazos con comunidades vecinas, respetando a los demás y no explotando los recursos naturales.

La ESS rechaza la actual lógica de mercado llena de intereses y egoísmo por parte de las competencias quienes buscan su engrandecimiento personal más no grupal, al contrario de la economía solidaria la cual busca una reciprocidad, redistribución y mercado para sus participantes.

\section{Economía Circular}

Gaztelumendi et al. (2019) señala que:

El modelo de producción y consumo que se ha venido desarrollando tradicionalmente responde a un sistema lineal en el que los recursos naturales son extraídos, ya sea para ser utilizados directamente o para ser transformados en bienes, los cuales son vendidos para ser usados durante un período de tiempo determinado y, finalmente, acaban siendo desechados, en un vertedero, generando grandes cantidades de residuos. (p. 54)

Desarrollar una economía circular en el turismo puede ayudar según Torán (2018) a "atraer el uso sostenible de los recursos, a mejorar la eficiencia en la industria turística y a alcanzar un desarrollo turístico sostenible" (p. 27).

Según la Ellen MacArthur Foundation (2013) (citado en Gutiérrez \& Carrillo, 2021), la economía circular es un sistema industrial que es restaurativo y regenerativo por intención y diseño. Reemplaza el concepto de 'fin de vida útil' por la restauración, se desplaza hacia el uso de energía renovable, elimina el uso de productos químicos tóxicos, que afectan a la reutilización, y tiene como objetivo la eliminación de los residuos a través de un diseño superior de materiales, productos, sistemas, y, con ellos, de los modelos de negocio.

El modelo de producción que las personas hemos ido utilizando a lo largo del tiempo responde a un sistema lineal tradicional, se trata de obtener recursos naturales y que estos sean transformados en bienes materiales para luego ser vendidos y desechados generando una gran cantidad de residuos.

La economía circular es un sistema restaurativo y regenerativo que busca la disminución de residuos tratando de reutilizar desechos para transformarlos en bienes materiales los cuales pueden ser reutilizados, también busca maneras de no afectar el medio ambiente 
como el uso de energía renovable, eliminar el uso de químicos y cualquier cosa que afecte el planeta.

\section{Economía}

Alburquerque (2018) afirma que la economía se ocupa del estudio de cómo la sociedad lleva a cabo las actividades orientadas a la atención de las necesidades de la población a través de la producción y distribución de los bienes y servicios generados para ello.

Según Jorge (2020) la economía ayuda a fijar los principios y las correspondientes normas de aplicación, destinadas a poner los recursos naturales, los medios de producción, el capital, el trabajo, la técnica y la mecánica de las relaciones humanas en función de la vida de la sociedad.

Para Proaño et al. (2021) la generación de divisas y el crecimiento económico basado en «nuevos sectores» con la creación de nuevos puestos de trabajo son dos de los potenciales efectos más importantes del desarrollo del sector turístico en una economía.

Es el estudio que se le da a una sociedad mediante sus actividades económicas y el uso de recursos disponibles, que busca satisfacer las necesidades de la población a través de la creación y elaboración de productos. La economía busca un crecimiento económico por parte de las personas para que un país pueda salir adelante. En el sector turístico la generación de divisas y los nuevos puestos de empleo que se habilitan gracias a las visitas turísticas hacen que la economía del país crezca enormemente.

\section{Aliados Estratégicos Económicos}

Para Cesar (2020) igualmente, es vital evaluar de manera crítica las oportunidades que tiene el sector en uso de ciencia, tecnología e innovación, de tal manera que se usen como aliados estratégicos del turismo y a los cambios en los patrones del consumidor final, es decir el turista (p. 10). los cuales están siendo financiados por aliados estratégicos, los cuales son: Operadoras turísticas, ONGs y embajadas. Se encuentran trabajando con un POA relativamente bajo de alrededor de 200.000 Bs aprox. Lo cual a causa de las reducciones terminaron sin presupuesto para proyectos, por lo que gestionaron con cooperación internacional, cuerpos diplomáticos, embajadas y ONGs (Ticona \& Villca, 2021, p. 90).

La observación participativa permitió identificar a los aliados estratégicos relacionados a la gestión de sitios arqueológicos de Lima Metropolitana, como son el Ministerio de Cultura, los Gobiernos Locales, la academia, el sector privado y la sociedad civil (Alania et al., 2021, p.27).

Para que el sector turístico siga en aumento y obtener más posibilidades de visitas extranjeras tenemos que estar actualizados y equipados con todos los avances que la humanidad vaya elaborando ya sea en el campo de la ciencia, tecnología e innovación esto nos brinda muchos beneficios a la hora de recibir visitantes que consumen este tipo de productos toda la tecnología y avances científicos es financiado por diferentes 
organizaciones, aliados estratégicos, embajadas, y entre otros. Los cuales buscan un fin común y es el crecimiento de la economía nacional y saben que con la adquisición de todas estas tecnologías lo conseguirán.

\section{Cámara De Turismo}

Menciona Pérez (2019), en la Ley de Cámaras de Turismo se establece que se constituirá una cámara de Turismo por provincia, cuyo domicilio será la capital provincial y en aquellos cantones que cuenten con vocación turística, es así como la primera, en el Ecuador, fue creada en el año de 1995 en la ciudad de Quito, provincia de Pichincha, siendo la máxima representante de la actividad turística de la provincia y del país (p.19). Para el Instituto Costarricense de Turismo (ICT) una cámara de turismo es una “organización sin fines de lucro, conformada por empresa-ríos turísticos y afines, qué unidos, buscan en la actividad turística una alternativa de desarrollo socio económico local" (Obando, 2019, p.7).

Es por esto por lo que el desarrollo turístico impulsado por la Cámara de Turismo implica superación económica de los socios afiliados, así como también el incremento nacional de flujo de efectivo al aportar y explotar los rincones turístico de la cuidad. A la vez que se cumplen con los objetivos empresariales de la misma mediante la ejecución y cumplimiento de proyectos (Lafebre \& Vivar, 2020).

Con la finalidad de sacar el máximo potencial de nuestros atractivos turísticos la cámara del turismo es una herramienta que ayuda a empresarios y micro empresarios a tener éxito en sus negocios gracias al incremento turístico, todos los socios que están afiliados a la cámara de turismo obtienen beneficios que generan un incremento económico en sus negocios, gracias a la planificación y organización de la misma, haciendo que los turistas obtengan un servicio de calidad y su experiencia sea plena y placentera.

\section{Tipos de cámara}

La Cámara de Comercio de Ambato es una institución gremial y empresarial creada el 18 de septiembre de 1928, época en que la industria y el comercio de Ambato, demostraban prosperidad en el concierto nacional de la producción y el intercambio, aparte de que la producción agrícola era también trascendencia y gran significado en el convivir nacional (Morales, 2019, p. 44).

En el sitio oficial de la "Cámara de industrias y producción de Tungurahua" se ha podido encontrar todas las sociedades dedicadas a la actividad de fabricación de calzado con fines de lucro (Quinapanta, 2020, p.29).

La Cámara de la Industria Automotriz del Ecuador (CINAE) concentra a todas estas empresas en una asociación que vela por los intereses del sector y es administrada por un directorio multi participativo de las empresas socias (Aguirre, 2020, p.34).

Mejoran el bienestar de la economía de nuestro país gracias a los programas que se desarrollan en conjunto con la comunidad siendo también los portavoces de las ideas de 
los ciudadanos y ayuda a verificar si estás ideas puedan ser beneficiosas para la comunidad y sus miembros, con la finalidad de fomentar y apoyar el emprendimiento de toda la ciudadanía, dónde el crecimiento empresarial no sea problema y los implicados obtengan todos los beneficios.

\section{Asociación de cámaras}

En el año 1955 se crea la Asociación Nacional de Bananeros del Ecuador (ANBE) con sede en Guayaquil, con la finalidad de dar atención y resolución a todos los problemas relativos a la producción, comercio e industrialización del banano (Homero et al., 2021).

Por otro lado, según la Asociación Ecuatoriana de Plásticos, en investigación realizada en marzo del 2018 en el Ecuador existe un amplio rango de crecimiento que puede tener la industria de productos plásticos (Medina, 2020).

La asociación de exportadores de flores, Expoflores, constituye un canal de cooperación técnica y de transmisión de información y capacitación para productores grandes, medianos y pequeños (Prado \& Gregory, 2020).

La unión hace la fuerza esto nos deja claro que trabajando en conjunto tendremos menos posibilidades de fracasar, esto lo aplica la asociación de cámaras dónde una organización u empresa se une con otras organizaciones de manera unánime esto será de gran beneficio para todos los miembros que está asociación representan ya que existe mayor posibilidad de resolución de problemas, expansión de mercado, menos competencia en el mercado, transferencia de información y muchos beneficios que está unión brinda.

\section{Metodología}

El método descriptivo, por medio de este método se pudo describir, detallar y analizar las actividades socioeconómicas del país y los aliados estratégicos del turismo, este método me permitió realizar consultas en tiempo real, recolectar datos, estudiar, analizar y obtener resultados significativos referentes al tema estudiado. El método cuantitativo a través de este método se logró estudiar, analizar los resultados y los demás aliados estratégicos brindándonos un conocimiento más amplio del tema usando datos detallados. Los resultados que obtuvimos fue la obtención porcentual a través de la encuesta que se realizó en línea dirigida a la población Babahoyense, obteniendo una respuesta de 160 personas realizando una serie de preguntas referentes a las actividades socioeconómicas turísticas del país. La investigación cualitativa permitió la interpretación de los resultados que se obtuvieron con la encuesta realizada, dando un aporte muy importante en mi proyecto, permitió obtener cierta información de los encuestados sobre el nivel de conocimiento que ellos poseen de las actividades socioeconómicas turísticas del país.

\section{Resultados}

Las actividades socio-económicas son la llave del progreso de una comunidad, cuando se detectan las posibilidades de emprenderlas, en la región del litoral estas oportunidades son repetitivas con emplazamientos gastronómicos insuperables en la población visitante, 
estas manifestaciones culturales son un portal incomparable de mejoramiento en la calidad de vida de una población organizada con frentes económicos diversificados de platos típicos y comida típica regional, las capacidades de inventiva comunitaria son incuestionables con las ingeniosas maneras de combinar la productividad agrícola de la zona y otros elementos propios de la región.

\section{Tablas 1}

Turismo gastronómico y acompañamiento Mintur

\begin{tabular}{|c|c|c|}
\hline Indicadores dominantes & $\begin{array}{c}\text { Porcentaje de aceptación } \\
\text { prioritaria }\end{array}$ & $\begin{array}{l}\text { Acciones procedimentales en } \\
\text { beneficio de la creatividad e } \\
\text { innovación }\end{array}$ \\
\hline El Turismo Gastronómico & $29.9 \%$ & $\begin{array}{l}\text { Financiamiento } \\
\text { microempresarial, para servicios } \\
\text { complementarios }\end{array}$ \\
\hline $\begin{array}{c}\text { Acompañamiento del Ministerio } \\
\text { de Turismo }\end{array}$ & $69.9 \%$ & $\begin{array}{c}\text { Incentivar talleres participativos } \\
\text { y capacitaciones en } \\
\text { hospitalidad, amabilidad }\end{array}$ \\
\hline
\end{tabular}

Existen organizaciones que representan al sector público y privado de la productividad nacional, que brindan asesoramiento continuo y permanente, atendiendo las iniciativas productivas de la población estas organizaciones como el Ministerio de Turismo del Ecuador, maneja una plataforma que colabora con la dinámica de digitalización competitiva en talleres y capacitaciones que van a la vanguardia de las tendencias y mega tendencias turísticas, reforzando las capacidades poblacionales con el acompañamiento definitorio en permanencia de estas posibilidades intelectuales de gobernanza comunitaria.

De acuerdo a los resultados obtenidos en la encuesta realizada se considera que unos de los principales aliados estratégicos en el desarrollo del turismo es el Ministerio de Turismo institución que lidera la actividad turística con proyectos de capacitación permanente y actualizada en temas proyectivos de desarrollo comunitario este sector en conjunto con la academia brinda asesoramiento, cierra la brecha de inversionistas nacionales e internacionales en con la productividad socio-económica de la región, reflejada en el incremento de plazas de trabajo en el sector, entre otras posibles soluciones se detectaron la amplitud de convenios con otras organizaciones inclusive de promoción.

\section{Conclusiones}

- El conjunto de aliados estratégicos está siendo actualizados dentro de la elaboración de servicios complementarios en producción de eficiencia socioeconómica de integración entre: empresa pública, empresa privada, y la academia que propone integrar funciones para ampliar las funciones de cooperación interinstitucional con proyectos emblemáticos de promoción de atractivos turísticos y el aumento de la empleabilidad de profesionales graduados en el área específica de desarrollo recreacional. 
- Los procesos organizacionales en pequeñas, medianas y grandes empresas a través del turismo es un hincapié de la necesidad en programar ayudas nacionales e internacionales con fondos destinados a países subdesarrollados o en vías de desarrollo con potenciales de iniciativas de crecimiento económico poblacional y surgimiento de la sostenibilidad urbana y rural de los ecosistemas integradores de productividad y desempeño profesional mancomunado, direccionando el trabajo hacia los grupos de atención prioritaria.

\section{Referencias bibliográficas}

Aguirre, J. (2020). El desafío de la cuarta revolución industrial como objetivo de competitividad de las industrias ensambladoras de vehículos en el Ecuador con visión al 2025. Titulo de Magister. Universidad Internacional del Ecuador, Quito.

Alania, J., Faustino, N. \& María, S. (2021). "Lineamientos para la Gestión de los Sitios Arqueológicos de Lima Metropolitana”. Tesis de Magister. Universidad Del Pacífico, Lima.

Alburquerque, F. (2018). Conceptos básicos de economía. Madrid: Orkestra-Instituto Vasco de Competitividad Fundación Deusto. Baptista, A. (febrero de 2020). Economía Social y Solidaria como contribución a un Desarrollo más justo. De Economía Social y Solidaria como Contribución a un Desarrollo más justo.

Dávila, L. R. (2020). Asdrúbal Baptista o el espíritu del mundo. Procesos Históricos, (38), 163.

Cesar, C. (2020). Turismo y competitividad integral: un camino en recorrido. Título de Especialista en Formulación, Evaluación Social y Económica de Proyectos. Universidad Católica De Colombia, Colombia.

Clavitea, F. (2017). La Demanda Turística y el Impacto Socioeconómico. Tesis De Licenciatura. Universidad Nacional Del Altiplano, Perú.

Coraggio, J. (agosto de 2020). Contribuciones De Consejeres. Obtenido de Contribuciones De Consejeres: https://www.argentina.gob.ar/sites/default/files/coraggio.pdf

Gaztelumendi, I., Tarí, A. \& Mora, D. (2019). Informe Sobre Economía circular aplicada al turismo. España: Segittur.

Gutiérrez, M. \& Carrillo, J. (2021). Economía Circular y Turismo. Tesis De Licenciatura. Universidad De Alcalá, España.

Guzmán, E. \& Maldonado, M. (2021). Impacto Socioeconómico de la pandemia covid19 en las familias e la ciudadela Colinas del Sur del Cantón Pasaje, año 2021. Tesis De Licenciatura. Universidad Técnica de Machala, Machala. 
Homero, G., Clara, C., Carrera, J. \& Sambonino, B. (2021). Afectación a las exportaciones de banano ecuatoriano a causa de la pandemia por el covid19. South Florida Journal of Development, Miami.

Jorge, V. (2020). Ingeniería económica. Guía del estudiante. Universidad Central Del Ecuador, Quito.

Lafebre, E., \& Vivar, D. (2020). "Nivel de Cumplimiento de las Obligaciones Tributarias de los socios afiliados en la Cámara Provincial de Turismo. Tesis De Licenciatura. Universidad Del Azuay, Cuenca.

Medina, E. (2020). Diagnóstico y propuesta de mejoramiento de los procesos de producción para la microempresa de la industria de productos. Tesis de Maestría. Universidad Andina Simón Bolívar, Quito.

Morales, K. (2019). Estrategias de comunicación para el posicionamiento de la cámara de comercio de Ambato. tesis de licenciatura. Universidad Técnica de Ambato. Ambato.

Obando, F. (2019). Las Cámaras de Turismo y su relevancia para el desarrollo turístico en las zonas rurales: el caso de Cattemi y Bijagua deUpala. revista ventana, 7.

Pérez, D. (2019). “Los Centros de información Turística y su aporte al desarrollo turístico del cantón Baños de Agua Santa. Tesis de Licenciatura. Universidad Técnica De Ambato, Ambato.

Piquinela, P. (2020). La Carpa de Economía Social y Solidaria: Aportes al análisis de los procesos de subjetivación. Scielo.

Prado, M. \& Gregory, V. (2020). Una Inserción Particular en el Comercio Agrícola Mundial: El Caso de la Rosa Ecuatoriana. Latin American Journal of Trade Policy, 55.

Proaño, G., López, C. \& Roberto, C. (2021). La situación turística actual de Ecuador y su incidencia en la reactivación económica. FIPCAEC, 32.

Quinapanta, R. (2020). "La inversión en maquinaria y la rentabilidad en las empresas de calzado asociadas a la Cámara de Industrias y Producción de Tungurahua”. Tesis de Ingeniero. Universidad Técnica de Ambato, Ambato.

Sosa, G. (2018). Impacto socio económico y el turismo rural. Tesis En Licenciatura. Universidad César Vallejo, Perú.

Ticona, H. \& Villca, S. (2021). Diseño del producto turístico artesanal en la Ciudad de la Paz "paseo andino". tesis de licenciatura. universidad mayor de san Andrés, Bolivia.

Torán, A. (2018). Economía Circular y Turismo. Tesis de Licenciatura. Universidad De Las Islas Baleares, España. 


\section{PARA CITAR EL ARTÍCULO INDEXADO.}

Rivera García, C., Izurieta Puente, M., \& Zuñiga Mayorga, J. (2021). Actividades socioeconómicas y los aliados estratégicos en el turismo, ciudad de Babahoyo - Los Ríos. ConcienciaDigital, $4(4.2)$,

112-123. https://doi.org/10.33262/concienciadigital.v4i4.2.1978

\section{¿Ciencia}

El artículo que se publica es de exclusiva responsabilidad de los autores y no necesariamente reflejan el pensamiento de la Revista Conciencia Digital.

El artículo queda en propiedad de la revista y, por tanto, su publicación parcial y/o total en otro medio tiene que ser autorizado por el director de la Revista Conciencia Digital.

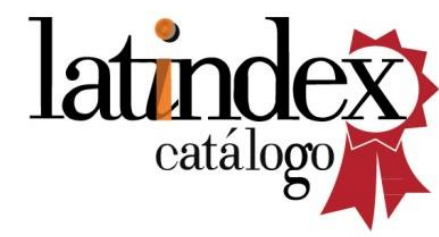

